\title{
Study of the wheel loader vibration with a developed multibody dynamic model
}

\author{
Nikolay Pavlov ${ }^{1, *}$, Evgeni Sokolov ${ }^{1}$, Mihail Dodov ${ }^{1}$, and Stoyan Stoyanov ${ }^{1}$ \\ ${ }^{1}$ Technical University - Sofia, Faculty of Transport, 8 Kliment Ohridski Blvd., 1000 Sofia, Bulgaria
}

\begin{abstract}
In this paper a multibody dynamic model of a compact wheel loader is created. This model includes the sprung and the unsprung masses of the tractor and the mass of the bucket. Results for the natural frequencies and the accelerations of the dynamic system are obtained. The dynamic model is verified by comparing the calculated results with the ground test results.
\end{abstract}

\section{Introduction}

Aggregating tractors with different agricultural implements (ploughs, tillers, sprayers, fertilizer spreaders) or construction machinery (excavators, hydraulic hammers, front-end loaders, etc.), results in an increase in their mass and moments of inertia. Their most negative impact is the increase of the mass moment of inertia around the lateral axis. It leads to a decreased frequency of oscillation around this axis and an increase in their amplitudes when the tractor is moving. As a result decreases ride comfort, which leads to fatigue in the driver-operator and a decrease in his ability to work.
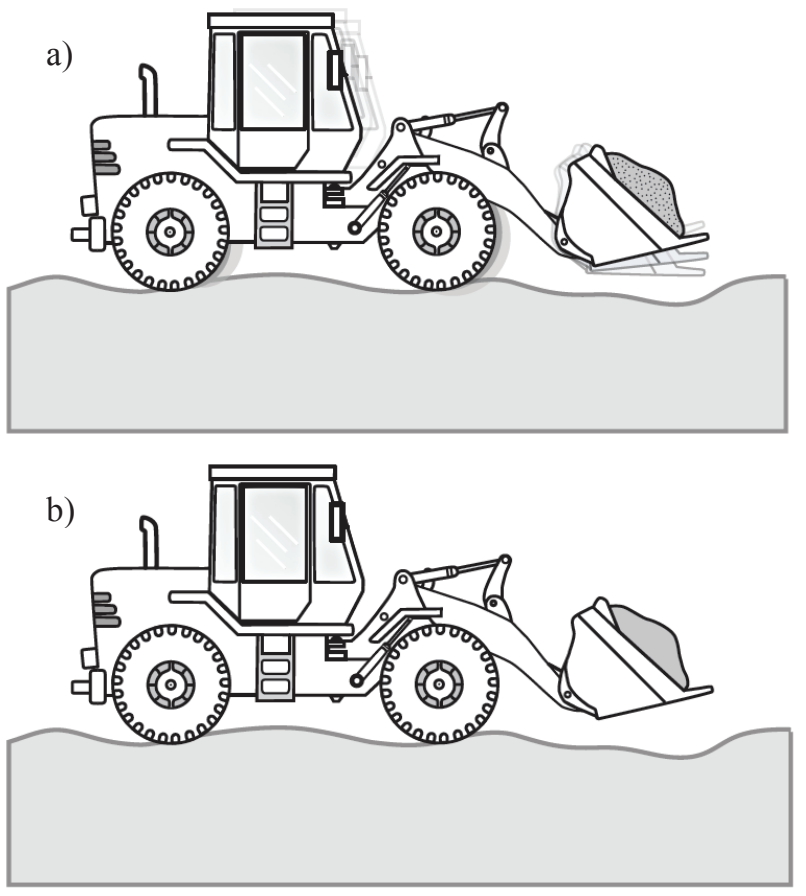

Fig. 1. Wheel loader moving on uneven road surface without boom suspension (a) and with boom suspension (b) [1].
In addition, there are major changes in the dynamic force of the front axle wheels, sometimes resulting in loss of contact with the road [1]. This leads to deterioration in handling the vehicle and traffic safety. If the movement is carried out with a load in the front loader bucket, angular oscillations with large amplitudes can result in the loss of part of the load (Fig. 1). Often, the operator has to decrease the travel speed to reduce the oscillations $[2,3]$.

Without an effective system for reducing the vibrations the efficiency, safety, and life of the machine can all be reduced, but most wheel loaders adopt rigid or semi-rigid suspension [4]. In order to improve the operation comfort of the wheel loader and reduce material falling out of the bucket, a type of vibration absorber system is created. To solve the problem, dynamic systems with hydraulic accumulators, operating as elastic elements and damping in hydraulic cylinders were proposed. The system is passive and requires a compromise solution when choosing constant coefficients of elasticity (the mass of the gas in accumulators is constant) and damping for different loads in the bucket of the loader. In order to fully solve the problem for all types of loading, more sophisticated mechatronic valve systems and pressure regulators in the hydraulic cylinders are used.

The purpose of this paper is to present five degrees of freedom (5DOF) dynamic model of a tractor front-end loader system. The model can be used for studying wheel loader vibration, ride comfort and road holding.

\section{Multibody model}

A multibody dynamic model of the system of a compact wheeled tractor with front-end loader was developed. Both axles of the tractor are elastic suspended. The model has five degrees of freedom. They are determinate of the vector of the generalized coordinates:

$$
q=\left[z_{0}, \theta_{0}, \theta_{f l}, z_{1}, z_{2}\right]^{T} .
$$

\footnotetext{
* Corresponding author: npavlov@,tu-sofia.bg
} 
A scheme of the model is shown in Fig. 2. This model includes the sprung mass (tractor body), the front and the rear unsprung masses (wheels and axles of the tractor) and the mass of the loader bucket.

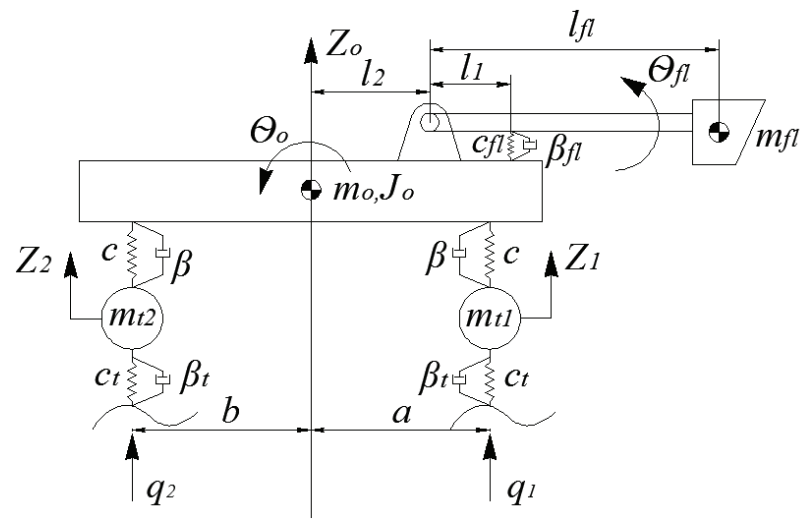

Fig. 2. Multibody dynamic model with five degrees of freedom.

The theoretical basis of the method is Lagrange equation of motion of the second kind:

$$
\frac{d}{d t}\left(\frac{\partial T}{\partial \dot{q}_{i}}\right)-\left(\frac{\partial T}{\partial q}\right)+\left(\frac{\partial U}{\partial q}\right)+\left(\frac{\partial R}{\partial \dot{q}}\right)=Q(t),
$$

where $T$ is the kinetic energy of the system; $U$ is the potential energy of the system; $R$ is Rayleigh dissipation function; $q_{i}$ are generalized coordinates; $Q(t)$ is function of kinematic disturbance.

The kinetic energy of the system is:

$T=\frac{1}{2} m_{0} \dot{z}_{0}^{2}+\frac{1}{2} J_{0} \dot{\theta}_{0}^{2}+\frac{1}{2} m_{f l} l_{f l}^{2} \dot{\theta}_{f l}^{2}+\frac{1}{2} m_{t 1} \dot{z}_{1}^{2}+\frac{1}{2} m_{t 2} \dot{z}_{2}^{2}$.

The potential energy of the system is:

$U=\frac{1}{2} c_{f l}\left(\theta_{f l} l_{1}-\theta_{0} l_{2}\right)^{2}+\frac{1}{2} c\left(z_{0}+\theta_{0} a-z_{1}\right)^{2}+\frac{1}{2} c\left(z_{0}-\right.$

$\left.\theta_{0} b-z_{2}\right)^{2}+\frac{1}{2} c_{t}\left(z_{1}-q_{1}\right)^{2}+\frac{1}{2} c_{t}\left(z_{2}-q_{2}\right)^{2}$.

The Rayleigh dissipative function is:

$R=\frac{1}{2} \beta_{f l}\left(\dot{\theta}_{f l} l_{1}-\dot{\theta}_{0} l_{2}\right)^{2}+\frac{1}{2} \beta\left(\dot{z}_{0}+\dot{\theta}_{0} a-\dot{z}_{1}\right)^{2}+\frac{1}{2} \beta\left(\dot{z}_{0}-\right.$ $\left.\dot{\theta}_{0} b-\dot{z}_{2}\right)^{2}+\frac{1}{2} \beta_{t}\left(\dot{z}_{1}-\dot{q}_{1}\right)^{2}+\frac{1}{2} \beta_{t}\left(\dot{z}_{2}-\dot{q}_{2}\right)^{2}$.

Their derivatives are:

$\frac{d}{d t}\left(\frac{\partial T}{\partial z_{0}}\right)=m_{0} \ddot{z}_{0} ;$

$\frac{d}{d t}\left(\frac{\partial T}{\partial \theta_{0}}\right)=J_{0} \ddot{\theta}_{0} ;$

$\frac{d}{d t}\left(\frac{\partial T}{\partial \theta_{f l}}\right)=J_{f l} \ddot{\theta}_{f l}=m_{f l} l_{f l}^{2} \ddot{\theta}_{f l} ;$

$\frac{d}{d t}\left(\frac{\partial T}{\partial z_{1}}\right)=m_{t 1} \ddot{z}_{1} ;$

$\frac{d}{d t}\left(\frac{\partial T}{\partial z_{2}}\right)=m_{t 2} \ddot{z}_{2} ;$

$$
\begin{aligned}
\frac{\partial U}{\partial z_{0}}=2 c z_{0}+c(a-b) \theta_{0}-c z_{1}-c z_{2} ; \\
\begin{array}{r}
\frac{\partial U}{\partial \theta_{0}}=c(a-b) z_{0}+\left(c_{f l} l_{2}^{2}+c a^{2}+c b^{2}\right) \theta_{0}- \\
c_{f l} l_{1} l_{2} \theta_{f l}-c a z_{1}+c b z_{2} ;
\end{array}
\end{aligned}
$$

$\frac{\partial U}{\partial \theta_{f l}}=l_{1}^{2} \theta_{f l}-c_{f l} l_{1} l_{2} \theta_{0} ;$

$\frac{\partial U}{\partial z_{1}}=-c z_{0}-c a \theta_{0}+\left(c+c_{t}\right) z_{1}-c_{t} q_{1} ;$

$\frac{\partial U}{\partial z_{2}}=-c z_{0}+c b \theta_{0}+\left(c+c_{t}\right) z_{2}-c_{t} q_{2} ;$

$\frac{\partial R}{\partial \dot{z}_{0}}=2 \beta \dot{z}_{0}+\beta(a-b) \dot{\theta}_{0}-\beta \dot{z}_{1}-\beta \dot{z}_{2} ;$

$\frac{\partial R}{\partial \dot{\theta}_{0}}=\beta(a-b) \dot{z}_{0}+\left(\beta_{f l} l_{2}^{2}+\beta a^{2}+\beta b^{2}\right) \dot{\theta}_{0}-$

$$
\beta_{f l} l_{1} l_{2} \dot{\theta}_{f l}-\beta a \dot{z}_{1}+\beta b \dot{z}_{2} ;
$$

$\frac{\partial R}{\partial \dot{\theta}_{f l}}=\beta_{f l} l_{1}^{2} \dot{\theta}_{f l}-\beta_{f l} l_{1} l_{2} \dot{\theta}_{0} ;$

$\frac{\partial R}{\partial \dot{z}_{1}}=-\beta \dot{z}_{0}-\beta a \dot{\theta}_{0}+\left(\beta+\beta_{t}\right) \dot{z}_{1}-\beta_{t} \dot{q}_{1} ;$

$\frac{\partial R}{\partial \dot{z}_{2}}=-\beta \dot{z}_{0}+\beta b \dot{\theta}_{0}+\left(\beta+\beta_{t}\right) \dot{z}_{2}-\beta_{t} \dot{q}_{2}$.

After applying the Lagrange equation (2) for equations describing the laws of motion of the system is valid:

$$
[M] \ddot{q}+[R] \dot{q}+[C] q=[Q],
$$

wherein $[M]$ is the matrix of inertia; $[C]$ is the stiffness matrix; $[R]$ is the Rayleigh's dissipative damping matrix.

They have the following form:

$$
M=\left[\begin{array}{ccccc}
m_{0} & 0 & 0 & 0 & 0 \\
0 & J_{0} & 0 & 0 & 0 \\
0 & 0 & m_{f l} l_{f l}^{2} & 0 & 0 \\
0 & 0 & 0 & m_{t 1} & 0 \\
0 & 0 & 0 & 0 & m_{t 2}
\end{array}\right]
$$

$\left[\begin{array}{ccccc}2 c & c(a-b) & 0 & -c & -c \\ c(a-b) & \left(c_{f l} l_{2}^{2}+c a^{2}+c b^{2}\right) & -c_{f l} l_{1} l_{2} & -c a & c b \\ 0 & -c_{f l} l_{1} l_{2} & c_{f l} l_{1}^{2} & 0 & 0 \\ -c & -c a & 0 & \left(c+c_{t}\right) & 0 \\ -c & c b & 0 & 0 & \left(c+c_{t}\right)\end{array}\right]$

$\left[\begin{array}{ccccc}2 \beta & \beta(a-b) & 0 & -\beta & -\beta \\ \beta(a-b) & \left(\beta_{f l} l_{2}^{2}+\beta a^{2}+\beta b^{2}\right) & -\beta_{f l} l_{1} l_{2} & -\beta a & \beta b \\ 0 & -\beta_{f l} l_{1} l_{2} & \beta_{f l} l_{1}^{2} & 0 & 0 \\ -\beta & -\beta a & 0 & \left(\beta+\beta_{t}\right) & 0 \\ -\beta & \beta b & 0 & 0 & \left(\beta+\beta_{t}\right)\end{array}\right]$.

$[Q]$ is the kinematic excitation vector.

\section{Numerical simulations}

The numerical simulations were performed in MATLAB with the parameters given in Table 1. 
Table 1. Model parameters.

\begin{tabular}{|c|c|c|}
\hline Symbol & Value & Unit \\
\hline$m_{0}$ & 1000 & $\mathrm{~kg}$ \\
\hline$J_{0}$ & 1000 & $\mathrm{Nm}^{2}$ \\
\hline$m_{f l}$ & 100 & $\mathrm{~kg}$ \\
\hline$m_{t 1}$ & 120 & $\mathrm{~kg}$ \\
\hline$m_{t 2}$ & 120 & $\mathrm{~kg}$ \\
\hline$c$ & 34000 & $\mathrm{~N} / \mathrm{m}$ \\
\hline$c_{t}$ & 160000 & $\mathrm{~N} / \mathrm{m}$ \\
\hline$c_{f l}$ & 42000 & $\mathrm{~N} / \mathrm{m}$ \\
\hline$\beta$ & 3000 & $\mathrm{Ns} / \mathrm{m}$ \\
\hline$\beta_{t}$ & 100 & $\mathrm{Ns} / \mathrm{m}$ \\
\hline$\beta_{f l}$ & 2000 & $\mathrm{Ns} / \mathrm{m}$ \\
\hline$a$ & 0,77 & $\mathrm{~m}$ \\
\hline$b$ & 0,43 & $\mathrm{~m}$ \\
\hline$l_{1}$ & 0,33 & $\mathrm{~m}$ \\
\hline$l_{2}$ & 1,07 & $\mathrm{~m}$ \\
\hline$l_{f l}$ & 0,9 & $\mathrm{~m}$ \\
\hline
\end{tabular}

The symbols used in Table 1 are:

$m_{0}, J_{0}$ - Sprung mass and moment of inertia; $m_{f l}-$ Front loader bucket mass;

$m_{t 1}, m_{t 2}-$ Front/rear unsprung mass;

$c, \beta$ - Suspension stiffness and damping;

$c_{t}, \beta_{t}$ - Tyre stiffness and damping;

$c_{f l}, \beta_{f l}$ - Boom suspension stiffness and damping;

$a$-Distance between front axle and tractor mass centre;

$b$ - Distance between rear axle and tractor mass centre;

$l_{1}$ - Distance between boom joint and boom suspension;

$l_{2}$ - Distance between boom joint and tractor mass centre;

$l_{f l}-$ Boom lenght.

To find the natural frequencies of the system, the equations are presented in Cauchy normal form [5]:

$$
\dot{y}+L y=0,
$$

where

$$
\begin{gathered}
y=\left[y_{1}, y_{2} \ldots y_{10}\right]^{T}=\left[q_{1}, q_{2}, \ldots q_{5}, \dot{q}_{1}, \ldots \dot{q}_{5}\right]^{T} ; \\
L=\left[\begin{array}{cc}
M^{-1} O & M^{-1} C \\
I & O
\end{array}\right]
\end{gathered}
$$

where $[I]$ is identity matrix and $[O]$ is zero matrix.

The natural frequencies with given in Table 1 parameters are:

$1,19 \mathrm{~Hz}$ - tractor vertical vibration $\left(z_{0}\right)$;

$0,5 \mathrm{~Hz}$ - tractor angular vibration $\left(\theta_{0}\right)$;

$1,72 \mathrm{~Hz}$ - front loader boom angular vibration $\left(\theta_{f l}\right)$; $6,4 \mathrm{~Hz}$ - wheel vertical vibration $\left(z_{1}, z_{2}\right)$.

To obtain the results for vibration of the system under sinusoidal disturbance, the equations are presented like:

$$
\dot{y}+L y=Y,
$$

where

$$
L=\left[\begin{array}{cc}
M^{-1} R & M^{-1} C \\
-I & O
\end{array}\right]
$$

$$
Y=\left[\begin{array}{c}
M^{-1} Q(t) \\
O
\end{array}\right]
$$

After integration of the equations using the RungeKutta method (ode45 solver in MATLAB), results for displacement, velocity and acceleration in a given time interval are obtained. Disturbing actions are applied in the contact patch centre of the tire with the road. They have the following form:

$$
q=q_{0}(1-\cos (v t)),
$$

where $q_{0}=0,04 \mathrm{~m}-$ amplitude of unevenness; $v-$ frequency of the disturbing action, $\mathrm{rad} / \mathrm{s}$.

A front-end loader with special construction was designed and made for verification of the dynamic model (Fig. 3).

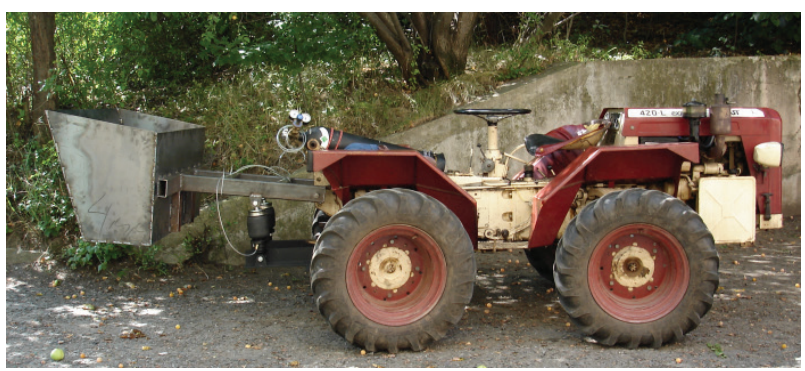

Fig. 3. Specially designed wheel loader.

The loader has pneumatic suspension to realise various stiffness ratios in the boom suspension and was attached to a compact wheeled tractor with suspended axles. Fig. 4 shows the ground test on special track with characteristics similar to the disturbances from the numerical experiment. An accelerometer for measuring acceleration was mounted on the driver seat (Fig. 5).

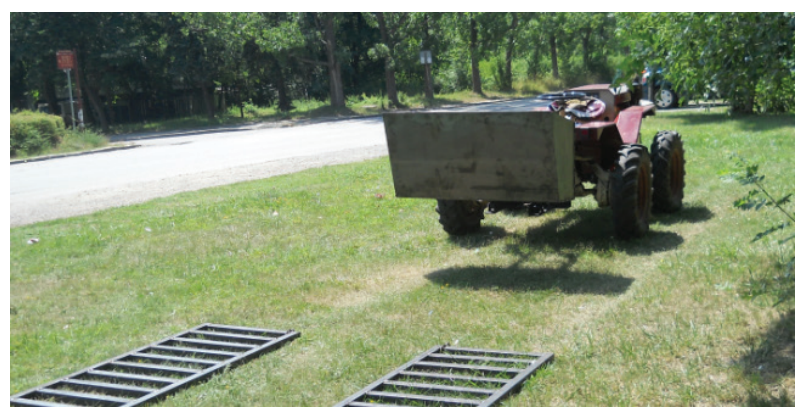

Fig. 4. Proving ground test on special track.

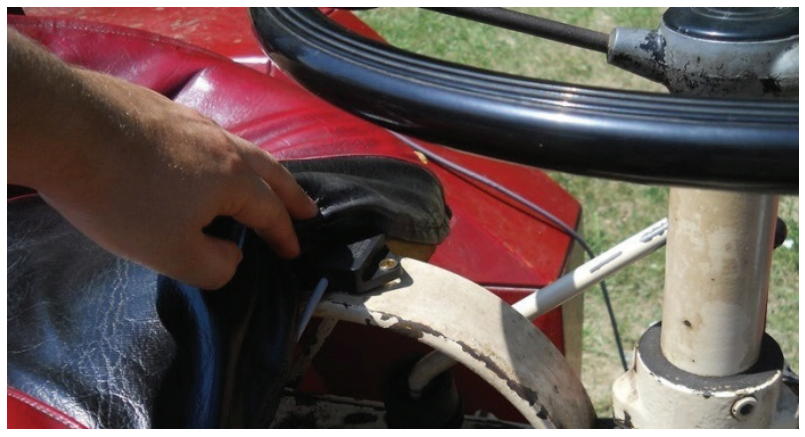

Fig. 5. Position of the accelerometer on the driver seat. 
A comparison of the calculated results and the ground test results for vertical acceleration on the seat can be made using Fig. 6 and Fig. 7.

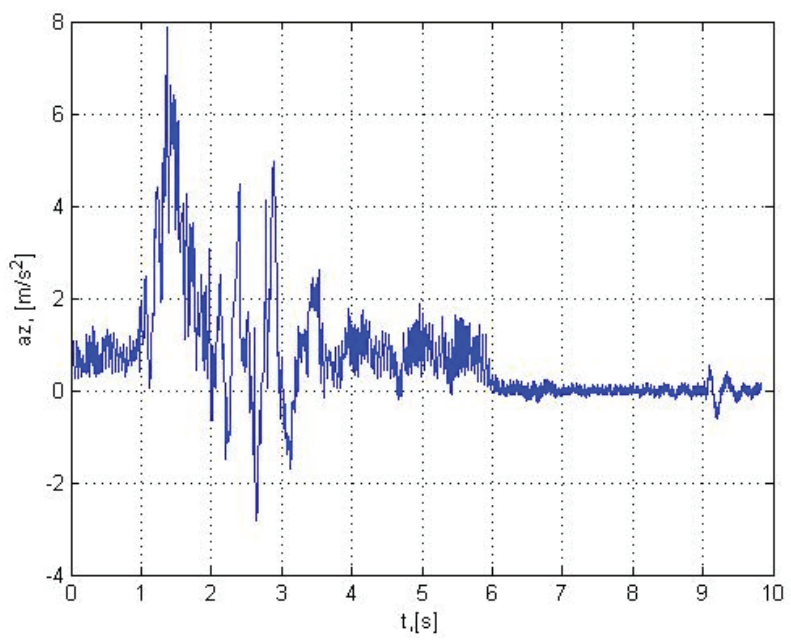

Fig. 6. Measured sprung mass vertical acceleration.

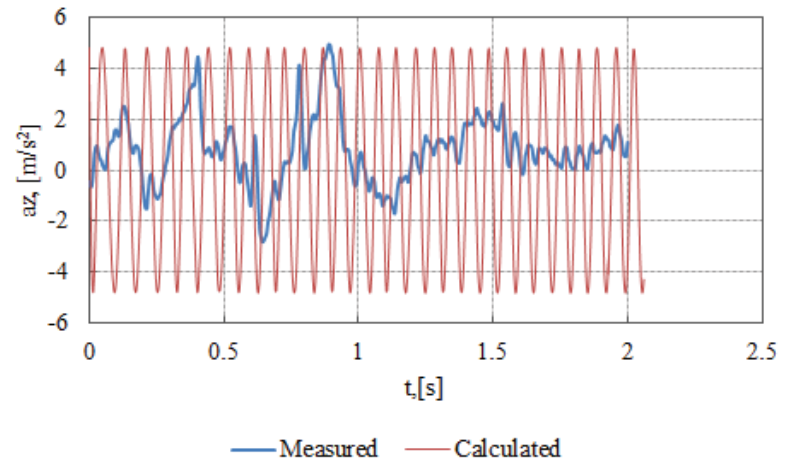

Fig. 7. Measured in time range of 2 to $4 \mathrm{~s}$ (the tractor moves on the track) and calculated sprung mass vertical acceleration.

The movement speed during the test was $8 \mathrm{~km} / \mathrm{h}$. The maximum values of the accelerations in the range of 2 to 4 seconds in Fig. 6 are commensurable with those obtained in the numerical experiments (Fig. 7). The asymmetry in measured acceleration comes probably from asymmetric characteristics of suspension shock absorbers which are not taken into account in the model. Before and after this time interval, the tractor practically does not move on the special test track. The maximum acceleration observed shortly after the 1-th second corresponds to the clutch engagement when the tractor begins to move. The acceleration between 4th and 6th second is obtained when the tractor moves on a meadow and after the 6-th second it is just from the engine force disturbance.

\section{Conclusion}

The developed multibody model makes it possible to study the vibration of sprung and unsprung masses, as well as the vibration of the loader boom. The model allows to study the vibrations at different stiffness and damping ratios of the boom suspension. The results may be used for estimation of dynamic properties related to wheel loader vibration, ride comfort and road holding.
The specially designed front loader allows for the realization of different stiffness ratios of the boom suspension. The front-end loader has been made and a ground test was conducted, which shows good comparability with the results from the numerical simulations.

The research presented in this paper was carried out with financial support from the Scientific and Research Sector of the Technical University of Sofia - Internal Funding Session 2017. Grant No. 171ПР0020-04.

\section{References}

1. http://www.hydraforce.com/Applications/WheelLoad er_solutions.html (Accessed 12 August 2017)

2. T. Langer, Comfort evaluation criteria for pitching vibration damping of agricultural tractors, Proceedings of LAND.TECHNIK, Germany, (2016)

3. R. Rahmfelda, M. Ivantysynova, An overview about active oscillation damping of mobile machine structure. Int. Journal of Fluid Power, 5(2), (2004)

4. Z. Wang, The simulation analysis and modelling of the coupling vibration absorber system for the wheel loader. Applied Mechanics and Materials, 121-126, 2358-2362, (2012)

5. M. Todorov, Study the vibration of forklift trucks and optimization of their parameters, $\mathrm{PhD}$ Thesis, Technical University - Sofia, in Bulgarian (1996) 